\title{
OS PRINCIPAIS FATORES INTERNOS E AS EXPORTAÇÕES MICRORREGIONAIS BRASILEIRAS*
}

\author{
Admir Antonio Betarelli Junior ${ }^{* *}$ \\ Eduardo Almeida ${ }^{* * *}$
}

RESUMO Este artigo analisa o comportamento das exportações como função da
disponibilidade da infraestrutura de transporte e do nível de PIB per capita das mi-
crorregiões brasileiras em 2003. Para esse propósito, foi implementada preliminar-
mente uma análise exploratória de dados espaciais e, em uma etapa posterior, de-
senvolveu-se uma análise econométrico-espacial por meio da inclusão da defasagem
espacial do termo de erro. Os resultados indicam que o crescimento econômico
contribui de forma significativa para o desempenho exportador das microrregiões.

Palavras-chave: exportações; transportes; análise exploratória de dados espaciais; econometria espacial

Código JEL: C21; R40

\section{THE INTERNAL FACTORS OF BRAZILIAN EXPORTS}

ABSTRACT The export growth plays an important role in the economic development and in the reduction of external vulnerability. This work analyzes the behavior the Brazilian exports in 2003 at the micro-regional level. Such an analysis evaluates the internal factors (GDP per capita and transport infrastructure) that influence the growth of the exports. To do so, an exploratory analysis of spatial data is preliminarily implemented. Further, spatial econometric analysis is developed by means of the inclusion of a spatially lagged of the error term. The main finding indicates that economic growth contributes significantly for the microregions'exporting performance.

Key words: exports; transportation; exploratory spatial data analysis; spatial econometrics.

\footnotetext{
* Artigo recebido em 26 de março de 2007 e aprovado em 8 de junho de 2009.

** Mestre em Economia Aplicada na Universidade Federal de Juiz de Fora e doutorando em Economia na Universidade Federal de Minas Gerais, e-mail: abetarelli@yahoo.com.br

*** Professor adjunto da Universidade Federal de Juiz de Fora e pesquisador do cnpq, e-mail: eduardo. almeida@ufjf.edu.br. Agradeço o apoio e financiamento do Conselho Nacional de Desenvolvimento Científico e Tecnológico (CNPQ), da Fundação de Amparo à Pesquisa do Estado de Minas Gerais (Fapemig) e da Universidade Federal de Juiz de Fora para o desenvolvimento deste artigo.
} 


\section{INTRODUÇÃO}

A partir de 1999, quando ocorreram a maxidesvalorização da moeda nacional e a mudança do regime cambial para flexível, as exportações passaram a ser um elemento-chave para reverter o deficit estrutural na conta de transações correntes do balanço de pagamentos (Baer, 1996). Desde então, o Brasil passou a contar com as suas exportações para tornar sua economia menos vulnerável externamente. Os aumentos sucessivos das exportações, neste período, corresponderam a ganhos de quase 10\% (2000) para o nível da demanda agregada brasileira. Ademais, conforme Young e Barbosa (1998), tais aumentos contribuíram para um desempenho superior das atividades exportadoras em relação às industriais tradicionais voltadas ao mercado interno.

De modo geral, observa-se que o desempenho exportador possui grande importância na determinação da situação econômica de um país, podendo, ser uma das alternativas para auxiliar no desenvolvimento econômico brasileiro (Haddad e Perobelli, 2002).

Como a economia brasileira não é internamente homogênea, os benefícios econômicos oriundos das exportações apresentam discrepâncias em nível microrregional. Haddad e Perobelli (2002) enfatizam que a heterogeneidade regional, representada "por questões estruturais do setor produtivo e tributárias, pela disponibilidade dos recursos naturais, incentivos governamentais, custos inerentes ao transporte e facilidade ao mercado externo, dentre outros", influencia o dinamismo regional exportador no comércio internacional.

Os fatores internos do Brasil, que geram margens fundamentais em termos de competitividade e diversificação da economia, tornam-se predominantes para averiguar o quanto se pode explicar do comportamento exportador regional frente às exigências cada vez maiores dos mercados globalizados e as crescentes integrações regionais.

Dentre os fatores internos Castro (1995) destaca a eficiência do sistema de transporte como condição básica para a competitividade de todos os setores da economia. Por outro lado, Azevedo et al. (2002) ressalta o fato de quanto maior for o PIB de uma nação, maior será a diversidade de mercadorias a serem ofertadas. Desse modo, o comportamento exportador será ava- 
liado pelo sistema de transporte e pelo PIB das microrregiões brasileiras (figuras 1 e 2).

A figura 1 apresenta a distribuição espacial do PIB per capita e as exportações por área das microrregiões brasileiras. Nessa figura verifica-se que as microrregiões que apresentam PIB per capita alto também têm um desempenho exportador significativo. Isso pode justificar a inclusão do PIB per capita como variável explicativa no modelo econométrico espacial. Além disso, é possível visualizar as desigualdades regionais brasileiras. Baer (1996) destaca que tais desigualdades podem ser reflexos do processo da industrialização ou do processo de substituição de importação que alterou a dinâmica de crescimento.

Já Castro (1999) salienta que a infraestrutura de transporte desempenha um papel relevante na determinação das decisões de produção, comércio e

\section{Figura 1: Distribuição espacial de variáveis selecionadas das microrregiões brasileiras (2003)}

Exportações

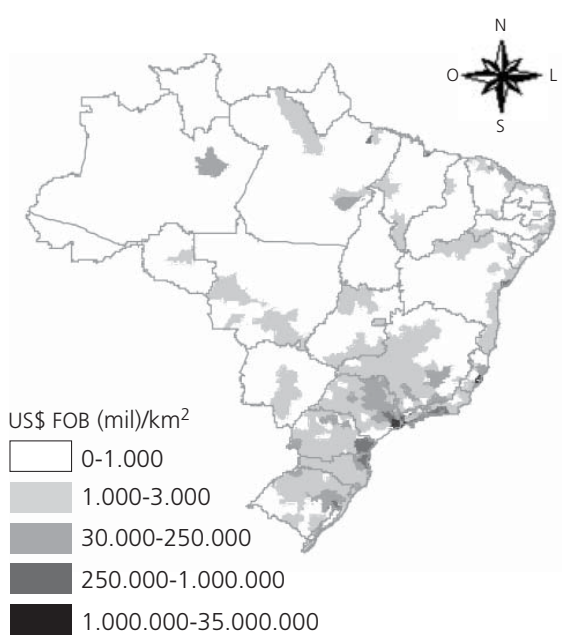

900

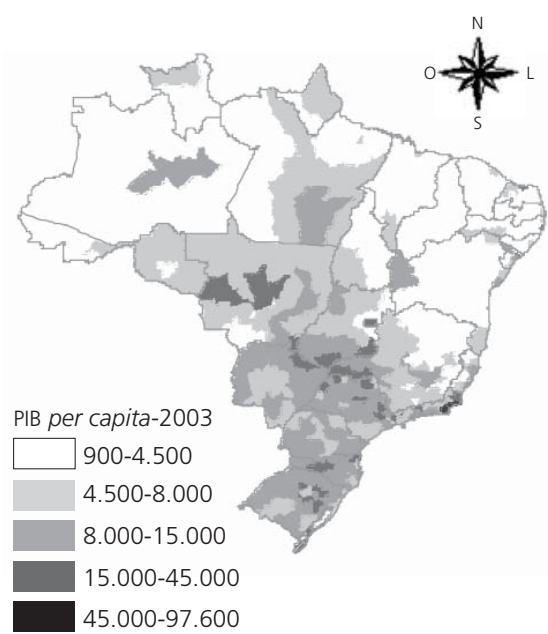

900

900

1.800 milhas

Fonte: IBGE (2006) — o arquivo shape e o dados da renda per capita; os valores (US\$ — FOB) das exportações — Ministério do Desenvolvimento da Indústria e Comércio (MDIC) — apud IPEAdata (2006). 


\section{Figura 2: Os principais sistemas de transporte e as exportações (2003)}

Sistema portuário

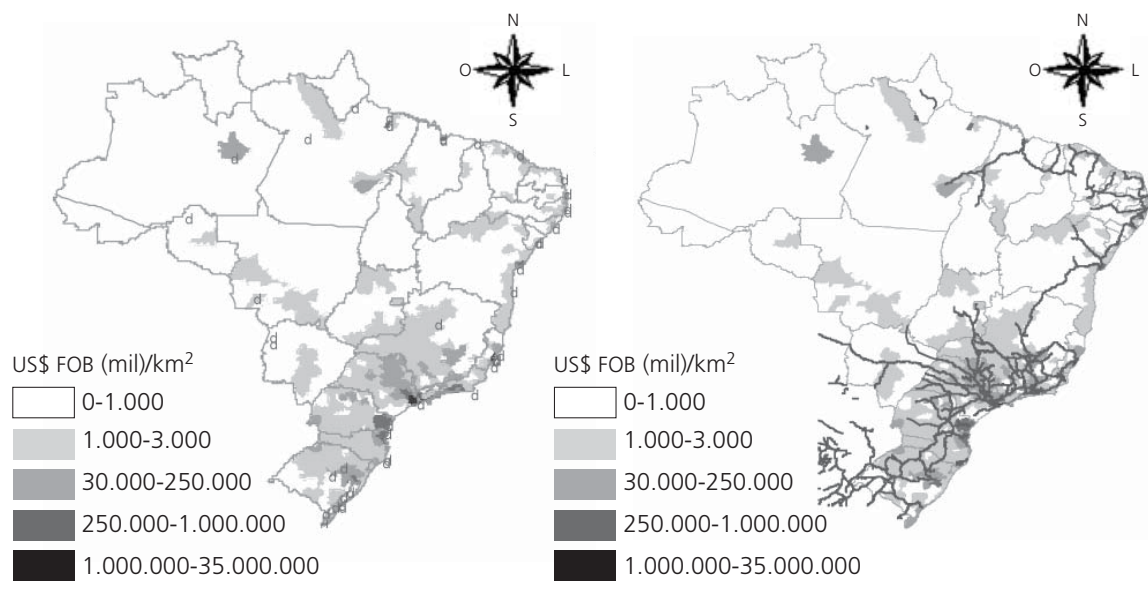

900
Rodovias pavimentadas

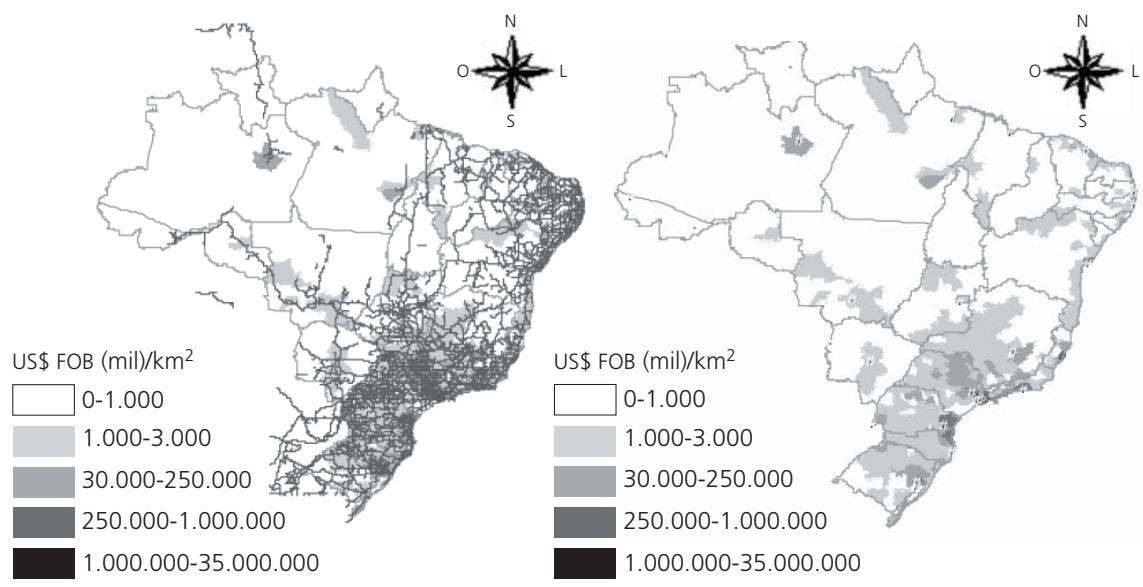

$\begin{array}{lllllll}900 & 0 & 1.000 & 2.000 \text { milhas } & 900 & 0 & 900\end{array}$ 
de consumo, assim como as decisões de localização e investimento por partes das firmas. ${ }^{1}$ Nesse sentido, a disponibilidade de infraestrutura de transporte pode condicionar o desempenho econômico (PIB) regional em virtude da concentração das indústrias. Por outro lado, o sistema de transporte pode ser condicionado justamente pela expansão da demanda devido ao aumento dos fluxos de pessoas e mercadorias. Não obstante, o comportamento exportador pode exercer uma relação similar com este tipo de infraestrutura. Como o desempenho exportador depende da disponibilidade e da capacidade de infraestrutura de transporte para o escoamento das mercadorias, a figura 2 ilustra os principais sistemas de transporte com as exportações microrregionais.

Na figura 2, observa-se que as microrregiões que apresentam alta exportação estão, na maioria, interceptadas pelas rodovias pavimentadas, ferrovias e pelo sistema portuário, como por exemplo, as microrregiões como São Paulo, Vitória, Guarulhos, Santos, São José dos Campos, Osasco, Salvador, Campinas, Itajaí, Rio de Janeiro, Porto Alegre e Belém. Isso pode indicar que a disponibilidade de infraestrutura pode condicionar o desempenho exportador microrregional ou ser condicionada justamente pela expansão da demanda devido ao aumento dos fluxos de mercadorias enviadas ao comércio internacional.

Apesar de os mapas citados indicarem uma possível relação positiva entre o PIB per capita e a maioria dos principais sistemas de transporte com as exportações por área das microrregiões brasileiras, é necessário verificar o grau de autocorrelação espacial dessas variáveis em questão. Para se conseguir isso, será utilizado um conjunto de técnicas de análise exploratória de dados espaciais (Aede) para posteriormente subsidiar os modelos econométrico-espaciais.

Além dessa seção introdutória, esse trabalho se organiza em mais 4 seções. A segunda seção apresenta o modelo teórico da econometria espacial. Em seguida, a terceira seção aborda a base de dados e a metodologia da Aede. A quarta seção trata a forma funcional do modelo empírico a ser estimado. Por fim, na quinta seção, são tecidas as considerações finais, salientando suas contribuições, principais resultados e os possíveis avanços e extensões desse trabalho. 


\section{MODELO TEÓRICO}

A econometria espacial é o subcampo da econometria preocupado em especificar, estimar e testar modelos, levando-se em conta a influência dos efeitos espaciais, a saber, dependência espacial e heterogeneidade espacial.

Os dois modelos espaciais mais frequentemente encontrados na literatura são o modelo de defasagem espacial e o modelo de erro espacial. No modelo de defasagem espacial, utiliza-se um termo para capturar um provável efeito de vizinhança, por exemplo, a cultura exportadora de uma dada região sendo imitada pelos seus vizinhos. Esse modelo pode ser expresso da seguinte forma:

$$
y=\rho W y+X \beta+\varepsilon
$$

em que $y$ é a variável dependente, $X$ é uma matriz composta por variáveis explicativas exógenas, $W y$ são a defasagem espacial para a variável dependente. Por sua vez, $\rho$ é coeficiente autorregressivo espacial (um escalar), enquanto que $\beta$ é um vetor de coeficientes.

Já no modelo de erro espacial, procura-se capturar efeitos nãomodelados que se manifesta no termo de erro inovacional. As mensurações dos efeitos não-modelados são difíceis, mas apresentam um padrão espacial, espraiando por todas as regiões. O modelo de erro espacial apresenta a seguinte expressão:

$$
\begin{aligned}
& y=X \beta+u \\
& u=\lambda W u+\varepsilon
\end{aligned}
$$

em que o coeficiente $\lambda$ é o parâmetro do erro autorregressivo espacial que acompanha a defasagem do erro, $W u$. Esse efeito decai ao longo da sua transmissão, significando que $|\lambda|<1$.

Com dados em corte cruzado, a metodologia da econometria espacial sugere que se siga o procedimento proposto por Florax et al. (2003):

(1) Estimar o modelo clássico de análise de regressão linear por Mínimos Quadrados Ordinários (MQO). 
(2) Testar a hipótese de ausência de autocorrelação espacial devido a uma defasagem ou a um erro por intermédio das estatísticas Multiplicador de Lagrange (ML) $\rho$ (defasagem espacial) e Multiplicador de Lagrange (ML) $\lambda$ (erro espacial).

(3) Caso ambos os testes não sejam significativos, a utilização do MQO é a mais apropriada. Caso contrário, é preciso seguir para o próximo passo.

(4) Caso ambos sejam significativos, estima-se o modelo apontado como o mais significante de acordo com as versões robustas desses testes, ou seja, Multiplicador de Lagrange Robusto $\left(\mathrm{ML}^{\star}\right) \rho$ (defasagem espacial) e Multiplicador de Lagrange Robusto $\left(\mathrm{ML}^{*}\right) \lambda$ (erro espacial). Dessa forma, se $\mathrm{ML}^{\star} \rho>\mathrm{ML}^{\star} \lambda$, utiliza-se o modelo com defasagem espacial como o mais apropriado. Caso $\mathrm{ML}^{\star} \rho<\mathrm{ML}^{\star} \lambda$, usa-se o modelo de erro autorregressivo como o mais apropriado. Se este passo não for satisfeito, é preciso seguir para o próximo.

(5) Se o teste $\mathrm{ML}^{\star} \rho$ é significativo e o $\mathrm{ML}^{\star} \lambda$ não, adote o modelo de defasagem espacial. Caso contrário, vá para o próximo.

(6) Se o teste $\mathrm{ML}^{\star} \lambda$ é significativo e o $\mathrm{ML}^{\star} \rho$ não, adote o modelo de erro espacial. Se este passo não for satisfeito, é necessário seguir para o próximo.

(7) Se ambos os testes $\mathrm{ML}^{\star} \rho$ e $\mathrm{ML}^{\star} \lambda$ forem significativos estatisticamente, é preciso utilizar o modelo de defasagem espacial com erro autorregressivo.

A matriz de peso espacial utilizada neste trabalho refere-se à matriz geográfica de contiguidade por convenção "Torre" $W_{i j}$ (rook). Assim, apenas as fronteiras físicas com extensão diferente de zero entre as regiões são levadas em conta (Lesage, 1999). Se as regiões partilham uma fronteira comum, logo elas são contíguas (valor unitário). Essa matriz é descrita da seguinte forma:

$$
W_{i j}=\left\{\begin{array}{l}
1 \text { se } i \text { e } j \text { são contíguos } \\
0 \text { se } i \text { e } j \text { não são contíguos }
\end{array}\right.
$$




\section{DADOS}

A variável dependente são as exportações (US\$ FOB (mil)) das 558 microrregiões brasileiras ${ }^{2}$ do ano 2003. Os dados foram coletados no Instituto de Pesquisa Econômica Aplicada (IPEAdata, 2006), tendo como fonte o Ministério do Desenvolvimento da Indústria e Comércio (MDIC). Inicialmente os dados referiam-se aos municípios brasileiros e foram agregados por microrregiões pelo software ArcView GIS 3.2. Após esta agregação, foi realizada a consistência dos dados.

É importante destacar que a variável foi dividida pela área total (quilômetros quadrados $-\mathrm{km}^{2}$ ) de cada microrregião. Tal procedimento está em consonância com o alerta de Anselin (2005, p. 9) de que se as variáveis não forem intensivas, os resultados podem ser enganosos porque os indicadores podem estar correlacionados com a área ou população total da localidade geográfica específica.

A escolha de intensificar as exportações por área total $\left(\mathrm{km}^{2}\right)$ não foi arbitrária. Esse indicador de intensidade geográfica capta melhor as desigualdades entre microrregiões brasileiras de tal forma que favorecerá uma análise comparativa.

No processo da estimação, as exportações microrregionais por área serão explicadas pelas seguintes variáveis independentes: (a) Produto Interno Bruto (PIB) per capita; (b) rodovias pavimentadas; (c) ferrovias; (d) portos e (e) aeroportos internacionais.

Os dados do PIB per capita das microrregiões brasileiras resultaram da agregação do PIB e da população (estimada) dos municípios brasileiros. Esses dados foram obtidos pelo Instituto Brasileiro de Geografia e Estatística (IBGE, 2006). Quanto ao procedimento dessa agregação, em primeiro lugar, converteu-se o PIB e a população estimada dos municípios em suas respectivas microrregiões para, posteriormente, realizar a razão entre essas variáveis (PIB pela população das microrregiões).

As rodovias pavimentadas por microrregiões foram estimadas a partir dos dados do Anuário Estatístico de 2003 da Agência Nacional de Transporte Terrestre (ANTT, 2006) e pelo arquivo digital no formato shape obtido pelo IBGE (2006). No procedimento de obtenção de dados sobre rodovias, em primeiro lugar, foram geradas 558 planilhas de arquivo de dados através do software ArcView GIS 3.2 e, posteriormente, agregadas em uma única 
planilha. Dessa planilha, foi possível classificar os dados em suas respectivas Unidades Federativas e, com isso, obter o share de cada microrregião. Em seguida, esse parcela foi aplicada nos dados das rodovias pavimentas estaduais disponíveis pela ANTT (2006).

Da mesma forma que as rodovias pavimentadas, a extensão ferroviária foi estimada pelo mesmo procedimento. Os dados foram coletados do Anuário Estatístico dos Transportes de 2001 da Empresa Brasileira de Planejamento de Transportes (Geipot, 2006).

Em relação aos portos brasileiros, os dados tiveram um tratamento diferente. Considerar essa variável como dummy poderia provocar distúrbios nas estimativas, visto que microrregiões como Vitória (3), Porto Alegre (3), Salvador (2), Belém (2), Linhares (2), Rio de Janeiro (2) e Paranaguá (2) apresentam uma quantidade de portos superior a um (1). Como o comportamento exportador depende da capacidade dessa infraestrutura, os dados dos portos foram considerados como proxy da divisão entre o total da movimentação (toneladas) de cargas $^{3}$ embarcadas e o total de embarcações nos cais públicos e terminais fornecidos pelo Anuário Estatístico de 2003 da Agência Nacional de Transportes Aquaviários (Antaq, 2006). Assim, os dados passaram a representar a capacidade do sistema portuário.

E, por fim, foi possível mapear a quantidade de aeroportos internacionais. No entanto, verificou-se que nenhuma microrregião apresentou um número superior a um (1). Essa variável será considerada como dummy nas estimações.

\subsection{Análise Exploratória de Dados Espaciais (Aede)}

A realização de uma análise exploratória dos dados espaciais (Aede) pode contribuir para indicar o modelo mais apropriado. Dessa forma, através desse conjunto de técnicas da Aede, é possível averiguar como as exportações brasileiras por área de cada microrregião estão distribuídas aleatoriamente ou não.

\subsubsection{Autocorrelação espacial global univariada}

A autocorrelação espacial pode ser calculada pelas de estatísticas: I de Moran, $c$ de Geary e $G$ de Getis-Ord. Todas essas estatísticas indicam se existe ou não, diante das suas peculiaridades, autocorrelação espacial do atributo empregado. 
A estatística $I$ de Moran é expressa da seguinte notação matricial:

$$
I=\frac{n}{S_{o}} \frac{\sum_{i} \sum_{j} w_{i j} z_{i} z_{j}}{\sum_{i=1}^{n} z_{i}^{2}}
$$

onde $z_{t}$ é o vetor de $n$ observações para uma variável normalizada. $W$ é a matriz de pesos espaciais em que os elementos $w_{i j}$ indicam a forma como a região $i$ está espacialmente conectada com a região $j$ e os elementos da sua diagonal principal são iguais a zero. O termo $S_{o}$ é um escalar igual à soma de todos os elementos de $W$.

O coeficiente $I$ de Moran tem um valor esperado de $-[1 /(n-1)]$. Assim, valores de $I$ de Moran que excedem o valor esperado indicam autocorrelação espacial positiva. Por outro lado, valores de I abaixo do valor esperado representam uma autocorrelação espacial negativa.

O I de Moran fornece esses três tipos de informação. O nível de significância provê a informação se os dados estão distribuídos aleatoriamente ou não. O sinal positivo da estatística $I$ de Moran, desde que significativo, indica que os dados estão concentrados através das regiões. O sinal negativo, por sua vez, indica a dispersão dos dados. A magnitude da estatística fornece a força da associação espacial. Quanto mais próximo de um, mais forte é a concentração; quanto mais próximo de -1 , mais dispersos estão os dados.

A estatística $c$ de Geary, por sua vez, também avalia a autocorrelação espacial global. Da mesma forma que o I de Moran, testa a aleatoriedade espacial. A fórmula dessa estatística é dada por:

$$
c=\frac{n-1}{2 \sum_{i} \sum_{j} w_{i j}} \frac{\sum_{i} \sum_{j} w_{i j}\left(y_{i}-y_{i}\right)^{2}}{\sum\left(y_{i}-\bar{y}\right)^{2}}
$$

onde $n$ é o número de regiões, $y_{i}$ é a variável de interesse, $\bar{y}$ é a média dessa variável e $W_{i j}$ é o elemento da matriz de peso espacial.

$\mathrm{O}$ valor de $c$ de Geary situa-se entre 0 e 2, e seu valor esperado é 1. Diferentemente do I de Moran, nesta estatística valores menores que o seu esperado indicam autocorrelação espacial positiva, enquanto valores maiores que 1 , representam autocorrelação espacial negativa. 
Por fim, a estatística $G$ de Getis-Ord tem uma peculiaridade diferente do $I$ de Moran. Um valor positivo da estatística $G$ indica que microrregiões de alto valor de exportação por área tendem a estar rodeadas por microrregiões vizinhas que também apresentam alta exportação. Por outro lado, um valor negativo significa que microrregiões de baixa exportação por área tendem a estar rodeadas por pequena exportação por área dos seus vizinhos.

Da mesma forma das estatísticas anteriores, a $G$ de Getis-Ord testa a ausência de dependência espacial. No entanto, sua significância é avaliada por meio do valor da estatística $Z$ padronizada.

Esta estatística é calculada a partir da expressão:

$$
G=\frac{\sum_{i} \sum_{j} w_{i j}(d) y_{i} y_{j}}{\sum_{i} \sum_{j} y_{i} y_{j}}
$$

onde $y_{i}$ e $y_{i}$ são a variável de interesse, respectivamente, da região $i$ e $j$, e $w_{i j}(\mathrm{~d})$ é o elemento da matriz geográfica de distância binária.

Os resultados destas estatísticas para os dados das exportações por área das microrregiões brasileiras convergiram para o mesmo sinal (tabela 1). A estatística $I$ de Moran evidenciou um coeficiente de autocorrelação espacial positiva, de magnitude 0,31 . Esse valor indica concentração espacial das exportações por área das microrregiões, ou seja, no geral, microrregiões de alta exportação por área estão rodeadas por microrregiões vizinhas que também apresentam alta exportação por área e/ou microrregiões de baixa exportação por área encontram-se rodeadas por vizinhos que apresentam comportamento exportador similar. Além disso, pelo teste estatístico é possível rejeitar a hipótese nula de ausência de correlação espacial, ou seja, o

Tabela 1: Teste de autocorrelação espacial global

\begin{tabular}{|c|c|c|c|c|c|c|}
\hline Estatística & Valor & $\begin{array}{c}\text { Matriz de peso } \\
\text { espacial }\end{array}$ & Média & Desvio padrão & Z-Value & Prob. \\
\hline I de Moran & 0,3070 & Torre & $-0,0020$ & 0,0260 & 11,8810 & 0,0000 \\
\hline C de Geary & 0,7630 & Torre & 1,0000 & 0,0276 & $-8,6004$ & 0,0000 \\
\hline G de Getis-Ord & 0,0791 & Torre & 0,0100 & 0,0051 & 13,5792 & 0,0000 \\
\hline
\end{tabular}


coeficiente $I$ de Moran é estatisticamente significativo sob o pressuposto da normalidade. Da mesma forma, a estatística $c$ de Geary $(0,762)$ também é estatisticamente significativa e indica autocorrelação espacial positiva. Por sua vez, o valor $Z$ da estatística $G$ é positivo, indicando que existem hot spots, ou seja, uma concentração dos dados de acordo com um padrão alto-alto.

\subsubsection{Diagrama de dispersão de Moran}

O diagrama de dispersão de Moran permite interpretar a estatística $I$ de Moran. Esse diagrama está dividido em quatro quadrantes: Alto-Alto (AA), Baixo-Baixo (BB), Alto-Baixo (AB) e Baixo-Alto (BA). E as microrregiões localizadas nos quadrantes AA e BB representam clusters de valores similares, enquanto nos demais quadrantes referem-se aos clusters de valores dissimilares.

No contexto univariado, o diagrama refere-se a uma representação do coeficiente de regressão e permite visualizar a correlação linear entre duas variáveis $z$ e $W z$ (são padronizadas) através de um gráfico. Dessa maneira, o coeficiente $I$ de Moran é a inclinação da curva de $W z$ contra $z$.

Na figura 3, observa-se uma concentração em torno da média nos quatro quadrantes [Alto-Alto (AA), Baixo-Alto (BA), Baixo-Baixo (BB) e Alto-

Figura 3: Diagrama de dispersão de Moran univariado

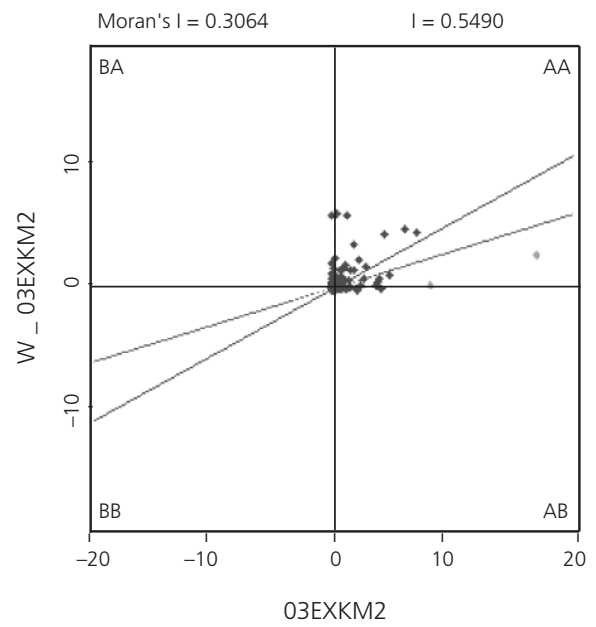


-Baixo ( $\mathrm{AB})$ ]. À primeira vista, há fortes indícios de que possa haver apenas outliers globais superiores. No quadrante Alto-Alto, como já era de esperar, as microrregiões com consideráveis desvios padrões são: São Paulo, Guarulhos, Santos, Osasco, Vitória e Rio de Janeiro.

Se excluir os chamados pontos de alavancagem (pontos cinzas claros na figura 3), ${ }^{4}$ como São Paulo e Vitória, o I de Moran atinge um maior valor $(0,55)$, indicando mais concentração espacial. As microrregiões que estão situadas no quadrante Baixo-Baixo são, na maioria, da região Norte, Nordeste e Centro-Oeste. Já nos outros quadrantes, é possível destacar as seguintes microrregiões: Salvador, Maceió, João Pessoa e Santa Tereza. Pelo mapa de dispersão de Moran (figura 4), podem-se visualizar essas regiões.

Figura 4: Mapa de dispersão de Moran
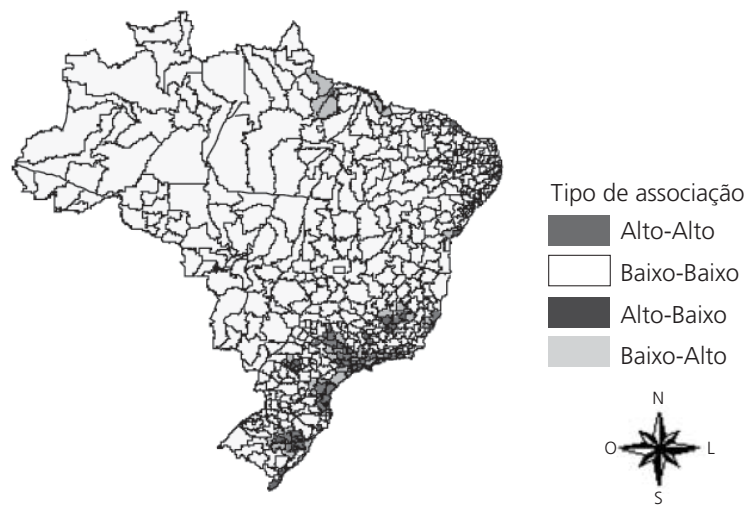

Já o diagrama de dispersão bivariado se baseia na estatística de $I$ de Moran bivariada (autocorrelação espacial bivariada). Entretanto, ao invés de utilizar a mesma variável das microrregiões, neste caso utilizam-se atributos diferentes. A ideia é analisar se os valores de uma variável observada em uma determinada região apresentam relação sistemática com os valores de uma outra variável observada em regiões vizinhas (Anselin et al., 2003).

Assim, a estatística de $I$ de Moran para duas variáveis diferentes ( $y$ e $x$ ) é dada por:

$I^{y x}=\frac{n}{\sum_{i} \sum_{j} w_{i j}} \frac{\sum_{i} \sum_{j}\left(x_{i}-\bar{x}\right) w_{i j}\left(y_{j}-\bar{y}\right)}{\sum_{i}\left(x_{i}-\bar{x}\right)^{2}}$ 
Da mesma forma que a estatística I de Moran univariada, os valores positivos e negativos dessa bivariada representam, respectivamente, concentração e dispersão espaciais. Entretanto, é necessário definir quais variáveis serão introduzidas nos eixos da abscissa e da ordenada. Assim, no eixo $x$, foram definidas as seguintes variáveis: renda per capita, densidade ferroviária, densidade rodoviária pavimentada e capacidade portuária por área. Por conseguinte, o eixo $y$ está representado pelas exportações por área das microrregiões. Com o intuito de reforçar as discussões realizadas na introdu-

Figura 5: Diagramas de dispersão de Moran bivariado, variáveis selecionadas
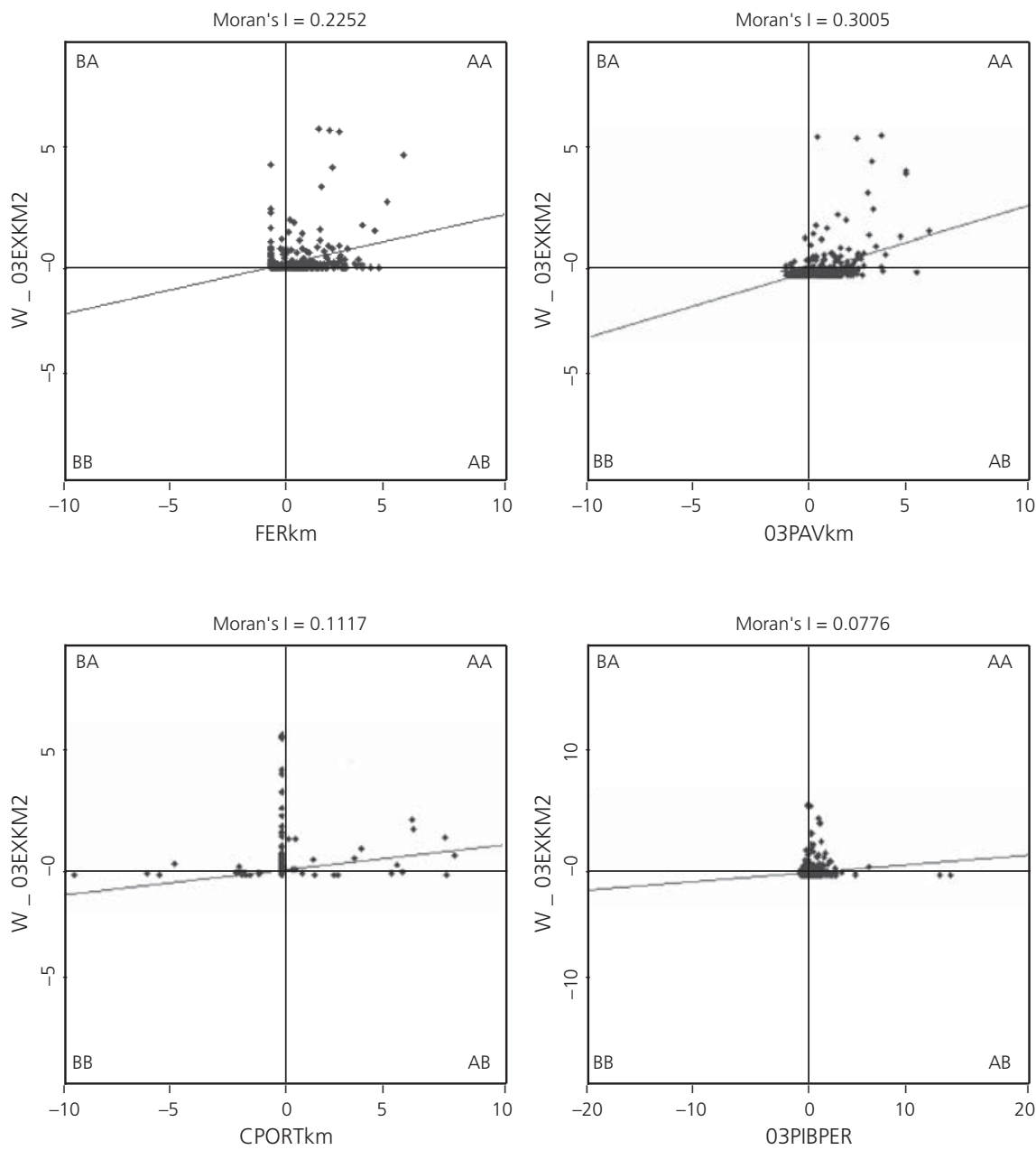
ção, os diagramas de dispersão bivariada da figura 5 destacam os valores positivos da estatística I de Moran.

Esses valores positivos descrevem que as microrregiões que apresentam exportação por área alta (baixa) tendem a estar rodeadas por microrregiões vizinhas com alta (baixa) renda per capita e disponibilidade das principais infraestruturas de transporte. Dito em outras palavras, os valores positivos apontam para uma similaridade no espaço entre as varáveis em questão.

Conforme esperado, a densidade de rodovias pavimentadas apresentou valor superior aos demais tipos de infraestrutura de transporte, embora todos sejam significativos em termos de magnitude.

\subsubsection{Autocorrelação espacial local}

Esse tipo de correlação espacial tem por objetivo captar padrões de associação local (clusters ou outliers espaciais) ocultados pelas estatísticas de autocorrelação global. Geralmente, isso ocorre em virtude das instabilidades locais presentes (Anselin, 1995).

A autocorrelação local pode ser calculada pela I de Moran local, que tem a capacidade de capturar padrões locais de autocorrelação espacial e estatisticamente significativos.

O coeficiente $I_{i}$ de Moran local para uma variável $y$ padronizada, observada na região $i, z_{i}$, pode ser expresso como:

$$
I_{i}=z_{i} \sum_{j=1}^{J} w_{i j} z_{j}
$$

No cômputo de $I_{i}$, só abrange os vizinhos da observação $i$, definidos conforme uma matriz de pesos espaciais.

Aplicando essa estatística para as exportações por área das microrregiões, observa-se, analisando o mapa de clusters obtido, que os resultados foram os esperados, inclusive em melhor acordo com o mapa de dispersão de Moran global (figura 4). Algumas microrregiões do Sudeste, situadas no quadrante Alto-Alto (clusters) de exportações por área, são estatisticamente significativas no nível de 5\%. Por outro lado, grande parte das microrregiões do Norte e Nordeste foi classificada como Baixo-Baixo (figura 6). 

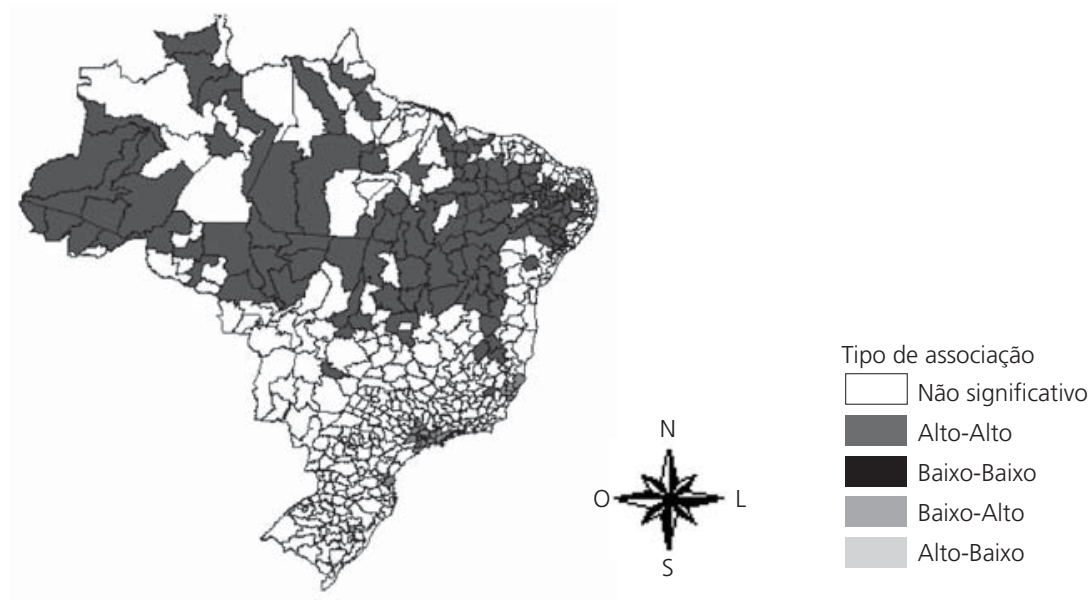

\subsubsection{Detecção de outliers globais e espaciais}

Da mesma forma que a tipologia da autocorrelação espacial, os outliers também são classificados entre naturezas globais e espaciais. O outlier global é definido como uma observação discrepante (superior ou inferior) dos restantes das outras observações. As estatísticas Gi ou $I_{i}$ podem ser usadas para identificar as microrregiões que são outliers globais. Além disso, existem outras formas de detectar os outilers globais, como: box plot, box map e cartograma.

$\mathrm{Na}$ figura 7, é interessante observar que as microrregiões consideradas outliers globais estão situadas na costa brasileira e/ou são as que se beneficiam de uma infraestrutura (rodovias, portos e ferrovias) instalada. Na figura 7, o tamanho de cada bola representa o valor da exportação por área de cada microrregião. As bolas cinzas mais escuras identificam outliers globais, enquanto os lugares vazios são regiões que não apresentam uma forte cultura exportadora.

Já os outliers espaciais, por sua vez, representam as observações que não seguem o mesmo processo de dependência espacial que a maioria dos dados segue. A sua identificação é importante, pois exercem influências espúrias sobre a medida global de autocorrelação espacial, podendo, assim, desfigurar a estimativa do teste. 
Figura 7: Cartograma

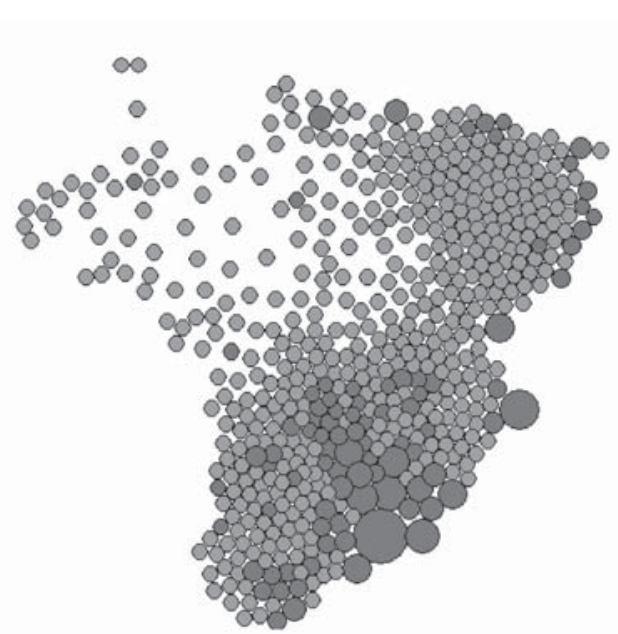

A detecção dos outliers espaciais é realizada através do diagrama de dispersão de Moran. Como visto, nesse diagrama existem quatro tipos de associações, ou seja, AA, BB, AB e BA. Uma indicação de autocorrelação espacial positiva (a inclinação da reta de regressão é positivamente inclinada) para uma determinada variável representa que a maioria das observações está localizada nos quadrantes AA e BB. Por outro lado, em uma autocorrelação espacial negativa, as observações situam-se nos quadrantes AB e BA (Anselin, 1996).

Dessa forma, diante de uma autocorrelação espacial positiva (por exemplo), os outliers espaciais serão aqueles localizados nos quadrantes AB e BA, enquanto as observações situadas nas associações AA e BB representam pontos de alavancagem. Esses pontos, por definição, são observações que exercem influências exageradas na determinação do grau de associação espacial, embora acompanhem a mesma associação espacial do restante dos dados (Anselin, 1996).

Tendo em vista que o diagrama de dispersão de Moran (figura 3, seção 2.2.2) indicou autocorrelação espacial positiva, os outliers espaciais são as microrregiões situadas nos quadrantes Baixo-Alto (BA) e Alto-Baixo (AB) com mais de dois desvios padrões da média. Nesse sentido, foram observado apenas três outliers espaciais inferiores, a saber: Paraibuna/Paraitinga (SP), Santa Teresa (ES) e Itanhaém (SP). 


\section{RESULTADOS E DISCUSSÕES}

O modelo empírico a ser estimado para as exportações por área das microrregiões brasileiras é definido como:

$$
\begin{gathered}
\ln \left(\exp / \mathrm{km}^{2}\right)_{i}=\alpha+\beta_{1} \ln (P I B / P O P)_{i}+\beta_{2} \ln \left(R O D P A V / \mathrm{km}^{2}\right)_{i}+ \\
\beta_{3} \ln \left[\left(\text { Ferr } / \mathrm{km}^{2}\right)^{*} 1000\right]_{i}+\beta_{4} \ln \left(\text { Cport } / \mathrm{km}^{2}\right)_{i}+\beta_{5} \text { Dae int }_{i}+\varepsilon_{i}
\end{gathered}
$$

onde

$\left(\exp / \mathrm{km}^{2}\right)_{i}$ representa a exportação por área da microrregião $i$;

$\alpha$ é o coeficiente fixo;

$(P I B / P O P)_{i}$ é o PIB per capita da microrregião $i$;

$\left(R O D P A V / \mathrm{km}^{2}\right)_{i}$ é a densidade de rodovias pavimentadas da microrregião $i$;

$\left[\left(\mathrm{Ferr} / \mathrm{km}^{2}\right)^{\star} 1000\right]_{i}$ é a densidade ferroviária multiplicada por 1000 da microrregião $i$;

$\left(\text { Cport } / \mathrm{km}^{2}\right)_{i}$ é a capacidade por área do porto da microrregião $i$;

Daeint $_{i}$ é a dummy, que possui valor unitário se $i$ tiver aeroportos internacionais, e zero caso contrário;

$\varepsilon_{i}$ é o erro aleatório.

A partir da forma funcional especificada, é importante tecer algumas considerações. Um problema comum que ocorre quando se utiliza o modelo com a especialização do tipo $\log$-log é o fato de as variáveis assumirem valores nulos (zero) na amostra. Como observado nas figuras 1 e 2, existem microrregiões que não apresentam cultura exportadora, rodovias pavimentadas, extensões ferroviárias e portos. Assim, devido à impossibilidade de obtermos o logaritmo de valores nulos (zero), inviabiliza a estimação pela forma funcional $\log$-log. Para a solução desse problema, existem duas sugestões conhecidas e práticas na literatura: (a) descartar as observações em que tal fato ocorre ou (b) substituir por valores próximos de zero. Como o contexto é espacial, não se podem simplesmente destacar microrregiões. Assim, será adotada, a sugestão (b). Além disso, os parâmetros estimados da forma funcional $\log$-log são entendidos como elasticidades, exceto para a variável dummy.

A partir da equação (8) é possível gerar as estimativas dos parâmetros das variáveis explicativas. Em um primeiro passo, conforme o procedimento descrito na primeira seção, deve-se, inicialmente estimar a regressão (08) por MQO. 
Tabela 2: Diagnóstico da regressão por MQO para as exportações por área das microrregiões

\begin{tabular}{lcc}
\hline Testes & Valor & Prob. \\
\hline Jarque-Bera & 13,89491 & 0,00096 \\
\hline Breusch-Pagan & 2,18331 & 0,82324 \\
\hline Koenker-Bassett & 2,41959 & 0,78855 \\
\hline Ide Moran & 10,15460 & 0,00000 \\
\hline Kelejian-Robinson & 92,74984 & 0,00000 \\
\hline$M L-$ error & 96,68621 & 0,00000 \\
\hline$M L^{*}-$ error & 43,07862 & 0,00000 \\
\hline$M L-$ defasagem & 56,65915 & 0,00000 \\
\hline ML* - defasagem & 3,05156 & 0,08066
\end{tabular}

A tabela 2 apresenta o primeiro diagnóstico da regressão por MQO. ${ }^{7}$ Nesta tabela observa-se que o teste de Jarque-Bera indica que os erros não seguem uma distribuição normal. Já pelos testes de Breusch-Pagan e KoenkerBassett, não se rejeita a hipótese de homocedasticidade. O modelo também não apresenta problemas de multicolinariedade. Os testes específicos de autocorrelação espacial, representados pelo multiplicador de Langrage (ML), foram, na sua maioria, estatisticamente significativos. A exceção fica por conta do teste robusto de defasagem espacial no nível de significância de $5 \%$. Os testes de autocorrelação espacial indicam o modelo de erro espacial como o mais apropriado.

No entanto, é preciso, antes de adotar o modelo de erro espacial, tentar corrigir a não normalidade dos erros. Dessa maneira, uma análise de possíveis outliers inferiores e superiores dos resíduos nessa regressão é necessária. Pelo mapa e pelo box plot de resíduos (figuras 8 e 9), é possível observar quais são as microrregiões que apresentam valores discrepantes (superiores ou inferiores) dos resíduos em relação às restantes observações.

Em uma análise comparativa entre as figuras 9 e 10, observa-se que existem apenas outliers globais inferiores. Esses são representados pelas regiões: Sergipana do Sertão do São Francisco, Bacia de São João, Contiguibá e Macaé. Dessa forma, para tentar resolver o problema da normalidade dos erros, será incluída uma variável dummy DINFR4. Nessa inclusão, as microrregiões verificadas receberão valor unitário e as demais, valores zeros. A tabela 3 reporta o diagnóstico da nova estimação por MQO. 


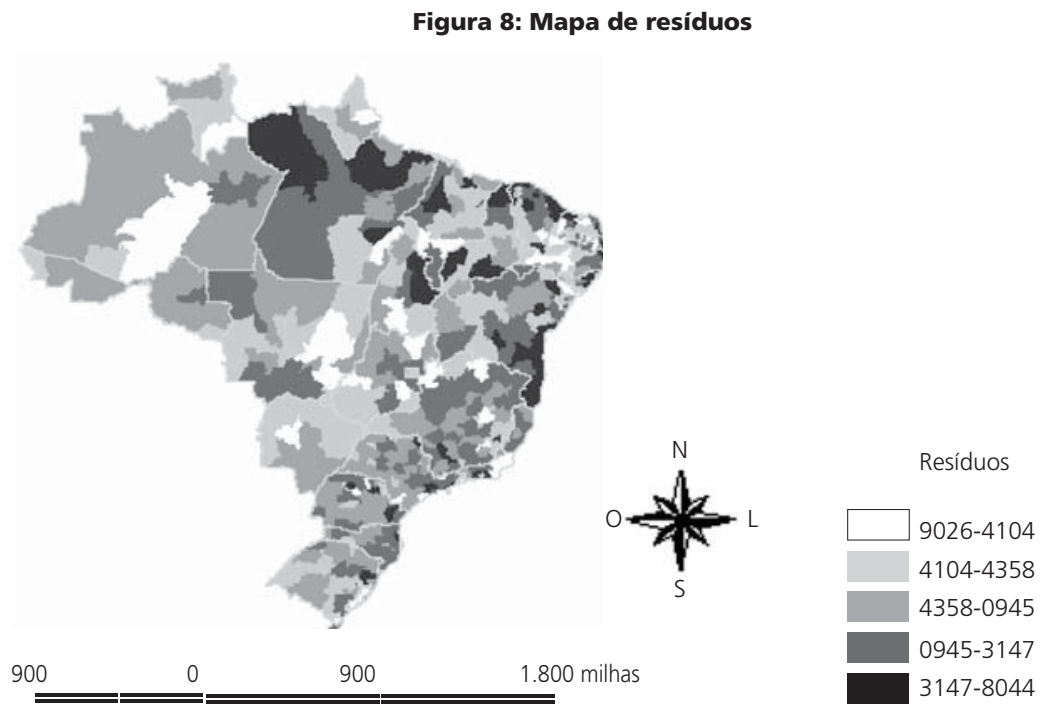

Figura 9: Box plot de resíduos

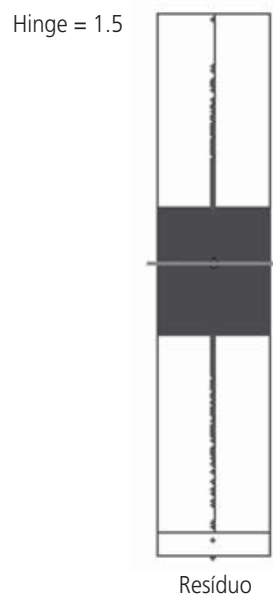

(558)

Pela observação da tabela 3, o valor da função verossimilhança ter aumentado (-1351.24), os critérios de Akaike (AIC) e Schwartz (SC) se reduzirem (respectivamente, 2716.48 e 2746.76). Os testes de Breusch-Pagan e Koenker-Bassett apresentam, agora, heterocedasticidade no nível de signifi- 
Tabela 3: Diagnóstico da regressão por MQO para as exportações por área das microrregiões

\begin{tabular}{lrl}
\hline Testes & Valor & Prob. \\
\hline Jarque-Bera & 8,19668 & 0,01660 \\
\hline Breusch-Pagan & 13,38843 & 0,03727 \\
\hline Koenker-Bassett & 15,74192 & 0,01571 \\
\hline Fonte: Elaboração própria através do programa Geoda &
\end{tabular}

Fonte: Elaboração própria através do programa Geoda.

cância de 5\%. E o teste de Jarque-Bera ainda acusa que os erros não são normais. Nessas circunstâncias, é necessário realizar alguns ajustes e testes na equação (8), ou seja, alternância de exclusão das variáveis e, em posterior, estimá-las por MQO.

Após uma bateria de ajustes entre variáveis, a equação que melhor apresentou resultados de estimativas pelo MQO é expressa da seguinte forma:

$$
\begin{aligned}
& \ln \left(\exp / \mathrm{km}^{2}\right)_{i}=\alpha+\beta_{1} \ln (P I B / P O P)_{i}+\beta_{2} \ln \left(R O D P A V / \mathrm{km}^{2}\right)_{i}+ \\
& \beta_{3} \ln \left[\left(\text { Ferr } / \mathrm{km}^{2}\right)^{\star} 1000\right]_{i}+\beta_{4} \ln \left(\text { Cport } / \mathrm{km}^{2}\right)_{i}+\beta_{5} \text { DINFR }_{i}+\varepsilon_{i}
\end{aligned}
$$

A exclusão da variável Dae int $_{i}$ gerou os seguintes resultados estimados por MQO, como reportados na tabela 4.

De acordo com a tabela 5 que reporta o diagnóstico dessa regressão, o valor da função verossimilhança aumentou (-1359.35), os critérios de Akaike (AIC) e Schwartz (SC) diminuíram (respectivamente, 2730.57 e 2756.52), e o valor da multicolinariedade reduziu (30.6306). O teste de Jarque-Bera indica que, no nível de significância de 5\%, os erros seguem uma distribuição normal. Já o teste de Breusch-Pagan acusa heterocedasticidade.

Tabela 4: Resultados da regressão por MQO para as exportações por área das microrregiões

\begin{tabular}{ccccc}
\hline Coeficientes & Valores & Desvio Padrão & t-value & Prob \\
\hline$\alpha$ & $-22,0653$ & 1,4411 & $-15,3119$ & 0,0000 \\
\hline$\beta_{1}$ & 3,2874 & 0,1683 & 19,5390 & 0,0000 \\
\hline$\beta_{2}$ & 0,1471 & 0,3686 & 3,9915 & 0,0001 \\
\hline$\beta_{3}$ & 0,4554 & 0,0855 & 5,3289 & 0,0000 \\
\hline$\beta_{4}$ & 0,5530 & 0,1863 & 2,9673 & 0,0031 \\
\hline$\beta_{5}$ & $-8,7857$ & 1,4228 & $-6,1748$ & 0,0000 \\
\hline
\end{tabular}


Tabela 5: Diagnóstico da regressão por MQO para as exportações por área das microrregiões

\begin{tabular}{lcl}
\hline Testes & Valor & Prob. \\
\hline Jarque-Bera & 5,826046 & 0,054311 \\
\hline Breusch-Pagan & 11,286905 & 0,045979 \\
\hline I de Moran & 9,594772 & 0,000000 \\
\hline Kelejian-Robinson & 91,104731 & 0,000000 \\
\hline ML - error & 85,856023 & 0,000000 \\
\hline$M L^{*}-$ error & 53,749468 & 0,000000 \\
\hline ML - defasagem & 32,120789 & 0,000000 \\
\hline ML* - defasagem & 0,014235 & 0,905029
\end{tabular}

Fonte: Elaboração própria através do programa SpaceStat.

Pelos testes de multiplicador de Lagrange, há a indicação de autocorrelação espacial na forma de erro.

Em consequência, a equação matemática do modelo de erro espacial é dada por:

$\ln \left(\exp / \mathrm{km}^{2}\right)_{i}=\alpha+\beta_{1} \ln (\mathrm{PIB} / \mathrm{POP})_{i}+\beta_{2} \ln \left(R O D P A V / \mathrm{km}^{2}\right)_{i}+$ $\beta_{3} \ln \left[\left(\text { Ferr } / \mathrm{km}^{2}\right) * 1000\right]_{i}+\beta_{4} \ln \left(\text { Cport } / \mathrm{km}^{2}\right)_{i}+\beta_{5}$ DINFR $_{i}+$ $(I-\lambda W)^{-1} \varepsilon_{i}$

O diagnóstico do modelo de erro espacial é reportado na tabela 6, indicando que não há remanescente autocorrelação espacial nos resíduos da regressão.

Neste modelo de erro espacial (tabela 6), verifica-se que os resultados foram os melhores entre todos os modelos estimados por MQO. O valor da função verossimilhança aumentou, ao passo que os dos critérios de Akaike (AIC) e Schwartz (SC) reduziram, e o resultado da multicolinariedade mostrou-se satisfatório. Os testes de Breusch-Pagan destacam a homocedastici-

Tabela 6: Diagnóstico da regressão por modelo de erro espacial para as exportações por área das microrregiões

\begin{tabular}{lll}
\hline Testes & Valor & Prob. \\
\hline Breusch-Pagan & 7,9234 & 0,1605 \\
\hline Spatial B-P & 7,9235 & 0,1605 \\
\hline Multplicador de Lagrange & 0,3367 & 0,5617 \\
\hline Fonte: Elaboração própria através do programa SpaceStat. &
\end{tabular}


dade do modelo. Nesse sentido, o problema de heterocedasticidade foi corrigido. Além disso, o teste do multiplicador de Lagrange indicou a ausência de dependência espacial dos erros.

$\mathrm{Na}$ forma funcional $\log$-log, os coeficientes estimados de cada variável são entendidos como elasticidades, exceto no caso da dummy. Assim, diante da tabela 7, observa-se que a elasticidade $\left(\beta_{1}\right)$ do PIB per capita das microrregiões é estatisticamente significativa. Da mesma forma, os coeficientes para as variáveis dos principais sistemas de transportes (densidade rodoviária pavimentada, extensão ferroviária por área e capacidade dos portos por área) foram estatisticamente significativos.

Em termos de magnitude, o coeficiente da variável PIB per capita foi o maior. Isso representa que o desempenho exportador microrregionais é mais elástico positivamente. Entre os coeficientes das principais infraestrutura de transporte, destaca-se a elasticidade $\left(\beta_{4}\right)$ da capacidade do sistema portuário, reforçando a análise realizada no cartograma (figura 7).

Diferentemente do esperado, o crescimento exportador é mais elástico para as extensões ferroviárias do que para as rodovias pavimentadas, o que reforça a importância de um melhor tratamento da infraestrutura ferroviária no Brasil. A interpretação é que um aumento de $1 \%$ na extensão ferroviária provoca uma elevação de $0,33 \%$ nas exportações por área, enquanto que um aumento de $1 \%$ em rodovias engendra um acréscimo de apenas 0,17\% nas exportações por área. É importante enfatizar algumas considerações sobre esta observação no resultado da regressão (tabela 7).

O setor do agronegócio, que detém baixo teor tecnológico, representa mais de $40 \%$ do total das exportações brasileiras em 2003. Assim, os fretes

Tabela 7: Resultados da regressão por modelo de erro espacial para as exportações por área das microrregiões

\begin{tabular}{crrrr}
\hline Coeficientes & Valores & Desvio padrão & t-value & Prob. \\
\hline$\alpha$ & $-22,0752$ & 1,8540 & $-11,9070$ & 0,0000 \\
\hline$\beta 1$ & 3,3192 & 0,2148 & 15,4515 & 0,0000 \\
\hline$\beta 2$ & 0,1668 & 0,0431 & 3,8740 & 0,0001 \\
\hline$\beta 3$ & 0,3333 & 0,0869 & 3,8341 & 0,0001 \\
\hline$\beta 4$ & 0,4849 & 0,1753 & 2,7659 & 0,0057 \\
\hline$\beta 5$ & $-7,1639$ & 1,3442 & $-5,3297$ & 0,0000 \\
\hline$\lambda$ & 0,0845 & 0,0093 & 9,1107 & 0,0000 \\
\hline
\end{tabular}

Fonte: Elaboração própria através do programa SpaceStat. 
oneram relativamente mais os preços finais desses produtos agrícolas. Entretanto, como que a cotação é fixada no mercado internacional, sendo difícil a transferência dos custos de transporte aos preços finais (Castro, 2003), as atividades desse setor devem, impreterivelmente, demandar um sistema de transporte que apresente um menor custo/distância. Castro (2000) destaca que o transporte de carga por rodovias tem um custo da ordem de $\mathrm{R}$ \$ 35-45 por 1.000 toneladas-quilômetro, enquanto as ferrovias brasileiras apresentam um custo de R\$15-26 por 1.000 toneladas-quilômetros. Ademais, no transporte de longa distância (acima de $1.000 \mathrm{~km}$ ), os custos ferroviários representam menos do que a metade dos custos rodoviários. Dessa maneira, o desempenho exportador em certas regiões pode ter sido condicionado pela disponibilidade de extensões ferroviárias.

Por fim, é interessante destacar que, pelo modelo de erro autorregressivo, as variáveis selecionadas explicam 51,5\% do comportamento exportador. Dessa forma, há indícios de que os demais 49\% são explicados por mais algumas variáveis classificadas como fatores internos. Um exemplo não considerado refere-se ao sistema de armazéns presente no Brasil. Esse sistema beneficia a logística de mercadorias agrícolas sensíveis (por exemplo, a temperatura) e perecíveis. A ausência desses armazéns pode prejudicar o escoamento dessas mercadorias até o mercado internacional, pois sua degradação obriga ao direcionamento para o mercado interno.

Esse hiato $(49 \%)$ pode também estar representado por variáveis de fatores externos. De qualquer forma, verifica-se que as culturas exportadoras das microrregiões são explicadas no mínimo, por 51\% do PIB per capita e a disponibilidade e capacidade da infraestrutura de transporte por área.

\section{CONSIDERAÇÕES FINAIS}

Neste artigo, procurou-se analisar o comportamento das exportações por área das microrregiões brasileiras, utilizando o conjunto de técnicas, instrumentos e procedimentos da econometria espacial.

Embora haja discrepâncias nas exportações devido à heterogeneidade microrregional, a análise confirma os resultados encontrados para os sinais e significância estatística dos coefeicientes das variáveis básicas desse tipo de modelo — PIB per capita, densidade rodoviária pavimentada, densidade ferroviária e capacidade dos portos por área. 
O trabalho também mostrou que um possível crescimento econômico per capita das microrregiões contribui significativamente para o aumento das suas exportações. Por outro lado, a capacidade e disponibilidade das principais infraestruturas de transporte também contribuem para impulsionar o desempenho exportador, com destaque para o sistema portuário.

Perante a importância do nível das exportações brasileiras para o equilíbrio do balanço de pagamentos e para a redução da vulnerabilidade externa do País, é patente a necessidade de manutenção e expansão desses fatores internos. Como visto, os fatores internos contribuem em 51\% para a explicação das exportações das microrregiões.

Além disso, embora esses resultados sejam promissores para as políticas econômicas, o presente trabalho apresenta limitações. Fatores específicos às regiões, mas não observáveis, tais como instituições, tecnologia e preferências, podem influenciar no desempenho econômico exportador. Trabalhos futuros precisam abordar tais fatores não observáveis, usando modelos de dados em painel com dependência espacial, a fim de aumentar a capacidade explicativa e preditiva do modelo. Longe de ser categórico, este trabalho apenas buscou evidenciar a importância dos referidos fatores internos para as exportações microrregionais brasileiras.

\section{NOTAS}

1. Essa ideia é similar às contribuições da Teoria da localização, a saber, Von Thünen (1826), Weber (1909), Lösch (1954), Isard (1951) e Moses (1958). Para maiores detalhes, consultar Ferreira (1989) e Figueiredo (1998).

2. O arquivo digitalizado no formato shape foi obtido no sítio eletrônico do IBGE.

3. Granéis sólidos, granéis líquidos e carga geral.

4. O conceito será visto na seção 2.2.4 (p. 216).

5. O valor da função verossimilhança foi de -1369.35 , os critérios de Akaike (AIC) e Schwartz (SC) foram, respectivamente, 2750,69 e 2776,64, e a condição de multicolinariedade registrou 30,62 .

\section{REFERÊNCIAS BIBLIOGRÁFICAS}

ALMEIDA, E. S; PEROBELLI, F. S; FERREIRA, P. G. C. Existe convergência espacial da produtividade agrícola no Brasil? [S.1.]: FEA/UFJF, 2006.

ANSELIN, L. Local indicators of spatial association — LISA. Geographical Analysis, n. 27, p. 93-115, 1995. 
Exploring Spatial Data with GeoDatm: a Workbook. Urbana-Champaign, University of Illinois: 2005.

The Moran scatterplot as an ESDA tool to assess local instability in spatial association. In: FISCHER, M.; SCHOLTEN, H.; UNWIN, D. Editors, Spatial Analytical Perspectives on GIS in Environmental and Socio-Economic Sciences. Londres: Taylor and Francis, 1996. p. 111-125.

; BERA, A. Spatial dependence in linear regression models with an introduction to spatial econometrics. In: ULLAH, A.; GILES, D. E. Editors, Handbook of Applied Economic Statistics. Nova York: Marcel Dekker, 1998. p. 237-289.

Spatial Econometrics: methods and models. Boston: Kluwer Academic, 1988. cap. 2. ; SYABRI, I.; KHO, Y. GeoDa: an introduction to spatial data analysis. [S.1.]: University of Illinois, 2003. (mimeo.)

AGÊNCIA NACIONAL DE TRANSPORTES AQUAVIÁRIOS (ANTAQ). Disponível em: <http:// www.antaq.gov.br/PortalPortos/EstatisticaAnuarios.htm> Acesso em 15 dez. 2006.

AGÊNCIA NACIONAL DE TRANSPORTE TERRESTRE (ANTT). Disponível em: < http://www. antt.gov.br/aett/FSCOMMAND/index.htm>. Acesso em 7 ago. 2006.

AZEVEDO, A. F. Z.; BARCELLOS, P. C. F.; PORTUGAL, M. S. Impactos comerciais da Área de livre comércio das Américas: uma aplicação do modelo gravitacional. UFRGS, 2002. < http:// www.ufrgs.br/ppge/pcientifica/2002_09.pdf>

BAER, W. A Economia Brasileira. São Paulo: Nobel, 1996. 416 p.

CASTRO, N. P. Custos de transporte e a estrutura espacial do comércio interestadual brasileiro. Pesquisa e Planejamento Econômico, Rio de Janeiro, v. 29, n. 3, 1999.

. Expansão rodoviária e desenvolvimento agrícola dos cerrados. In: HELFAND S.; REZENDE G. (Org). Região e Espaço no Desenvolvimento Agrícola Brasileiro. 1. ed. Rio de Janeiro: IPEA, 2003, v. 1, p. 2.

Intermodalidade, intramodalidade e o transporte de longa distância no Brasil. IPEA. Texto para Discussão, Rio de Janeiro, n. 367, 1995.

Os Desafios da Regulação do Setor de Transporte no Brasil. Revista Brasileira de Administração Pública, Rio de Janeiro, v. 34, n. 5, p. 119-141, set./out. 2000.

DE NEGRI, F. Conteúdo tecnológico do comércio exterior brasileiro: o papel das empresas estrangeiras. Texto para Discussão, Rio de Janeiro: IPEA, n. 1.074, 2005.

EMPRESA BRASILEIRA DE PLANEJAMENTO DE TRANSPORTES (GEIPOT). Anuário Estatístico dos Transportes. Disponível em: <http://www.geipot.gov.br/NovaWeb/IndexAnuario. htm> Acesso em 9 out. 2006.

FAIR, M. L.; WILLIAMS JR., E. W. Economics of transportation. Nova York: Harper \& Brother Publishers, 1959. 684p.

FERREIRA, C. M. As teorias da localização e a organização espacial da economia. In: Haddad, P. R. (Org.). Economia Regional: teorias e métodos de análise. Fortaleza: BNB, 1989 MDR. 
FIGUEIREDO, A. T. L. Padrão locacional e especialização regional da indústria mineira. 1998. cap. I. (Dissertação de Mestrado em Economia) — Universidade Federal de Minas Gerais (UFMG). Belo Horizonte, 1998.

FLORAX, R. J. G. M.; FOLMER, H.; REY, S. J. Specification searches in spatial econometrics: the relevance of Hendry's methodology. Regional Science and Urban economics, v. 33, n. 5, p. 557-79, 2003.

HADDAD, E. A.; PEROBELLI, F. S. Integração regional e padrão de comércio dos estados brasileiros, unidade e fragmentação: a questão regional no Brasil. Perspectiva, n. 284, p. 221 a 236, 2002.

INSTITUTO BRASILEIRO DE GEOGRAFIA E ESTATÍsTICA (IBGE). Base de Dados. Disponível em: <http://www.ibge.gov.br> Acesso em: 20 ago. 2006.

INSTITUTO DE PESQUISA ECONÔMICA APLICADA. BASE DE DADOS (IPEADATA). Disponível em: <http://www.ipeadata.gov.br> Acesso em 9 set. 2006.

ISARD, W. Distance inputs and the space economy: the locational equilibrium of the firm. Quarterly Journal of Economics. Cambridge, Mass., Harvard University, 1951.

LESAGE, J. P. Spatial econometrics. [S.1.]: Departamento de Economia. University of Toledo, 1999. (mimeo.) cap. 1.

LÖSCH, A. The economics of location. Yale: Yale University, 1954.

MARTINS, R. S.; CAIXETA FILHO, J. V. Evolução histórica da gestão logística do transporte de cargas. In: MARTINS, R. S.; CAIXETA-FILHO, J. V. (Org.). Gestão logística do transporte de cargas. 1. ed. São Paulo: Atlas, 2001. p. 15-31.

MOSES, L. N. Location and the theory of production. The Quartely Journal of Economics, Cambridge, Mas.: Harverd University, 1958.

VON THÜNEN, H. Der isolierte Staat in Beziehung auf Landwirtshaft und Nationalekonomie. Hamburg:[s.n.] 1826. v. 1.

WEBER, A. The theory of the location of industries. Chicago: University of Chicago, 1929.

YOUNG, C. E. F. ; BARBOSA FILHO, F. H. Comércio Internacional, Política Econômica e Poluição no Brasil. In: ENCONTRO NACIONAL DE ECONOMIA, VITÓRIA, 26. 1998. Anais, v. 3 , p. $1.573-1584,1998$. 